\title{
Accenting and detection of timing variations in tone sequences: Different kinds of accents have different effects
}

\author{
HASAN GÜRKAN TEKMAN \\ Middle East Technical University, Ankara, Turkey
}

\begin{abstract}
The effect of intensity and pitch accents on the perception of timing was examined in two experiments using a signal detection procedure. Analyses of sensitivity and response bias revealed opposite effects of intensity and pitch accents under similar conditions. Time intervals preceding intensity accents were perceived as longer, but time intervals preceding pitch accents were perceived as shorter. These results showed that listeners found it easier to detect timing variations that were contrary to expectations, as compared with variations that were consistent with expectations. In the present case, listeners should have expected shorter time intervals before intensity accents and longer intervals before pitch accents. The fact that the effects were observed with stimuli that had minimal musical structure demonstrated the contribution of psychoacoustic factors to such phenomena.
\end{abstract}

Research has shown that the detection of temporal irregularity in musical sequences of sounds is closely related to the structure of the sequence. The experiments reported in this article tested predictions from two alternative hypotheses that could explain the perceptual biases observed in such cases. One of these hypotheses will be called the pattern completion hypothesis. According to this hypothesis, listeners perceptually distort stimuli so that their perception is consistent with what they expect to hear (Thorpe \& Trehub, 1989; Thorpe, Trehub, Morrongiello, \& Bull, 1988; Woodrow, 1909). That is, they perceive illusory deviations from temporal regularity in the expected direction. The second hypothesis will be called the compensation hypothesis. According to this hypothesis, perception is distorted in the opposite direction to what is expected (Repp, 1992b, 1995, 1998). That is, listeners perceive illusory deviations in the opposite of the expected direction.

These hypotheses make opposite predictions about how musical sequences with totally regular timing would be perceived. A sequence of sounds with perfectly equal beat periods can still have variations in other properties of sound, such as pitch, intensity, duration, and harmonic function. Such variations would create specific expectations in terms of timing, because expressive temporal variations in actual performance are correlated with other as-

Parts of the research reported were presented at the 4th International Conference on Music Perception and Cognition, held in Montreal in August 1996, and the 5th International Conference on Music Perception and Cognition, held in Seoul in August 1998. The author thanks Bruno Repp, Carolyn Drake, and Arthur Samuel for their comments on an earlier version of the manuscript. Correspondence should be addressed to H. G. Tekman, Program in Cognitive Science, School of Informatics, Middle East Technical University, ODTU 06531, Ankara, Turkey (e-mail: tekman@metu.edu.tr). pects of musical structure (Penel \& Drake, 1998; Repp, 1990, 1992b, 1995, 1996, 1998; Shaffer, Clarke, \& Todd, 1985; Todd, 1985; Windsor \& Clarke, 1997). One very simple and general expectation is that consecutive sounds that belong to different perceptual groups should be separated by longer time intervals, as compared with consecutive sounds that belong to the same perceptual group (Handel, 1989, pp. 386-389). According to the pattern completion hypothesis, in such a sequence the temporal separations between perceptual groups should be perceived as longer, just as they are expected to be. According to the compensation hypothesis, just the opposite should happen. The time intervals that are expected to be longer should be heard as shorter, if they are equal to other time intervals and the expectation is not fulfilled. Thus, if listeners were trying to detect timing variations in such a sequence, the two hypotheses predict asymmetries in opposite directions for false alarm rates (i.e., the rate of reporting that an interval is different when it is in fact the same). According to the pattern completion hypothesis, listeners should make many false alarms reporting a variation in the expected direction but few false alarms reporting a variation in the unexpected direction. According to the compensation hypothesis, on the other hand, false alarms for reporting unexpected variations should be much more likely than those for reporting expected variations.

The two hypotheses also lead to opposite predictions if we consider sequences in which a single beat period is altered in the expected or the unexpected direction. According to the pattern completion hypothesis, a small change in the expected direction should be easy to notice (i.e., the hit rate should be high). This is because an actual variation of this kind would be added to the illusory variation that listeners are likely to hear. A change in the opposite direction to what is expected, in contrast, would be difficult to detect. This is because the actual timing 
variation would cancel out the illusory variation that is in the expected direction. This would create a sense of uniform timing. The predictions of the compensation hypothesis would be just the opposite of these. A timing variation in the expected direction should be difficult to detect, because it exactly fulfills an expectation. On the other hand, a variation in the unexpected direction would strongly violate an expectation. This violation of expectancy would be especially noticeable.

\section{Ways of Manipulating Expectancy}

In one line of research, Repp has demonstrated, with real music (Repp, 1992b, 1998) and with short melodies created for experimental purposes (Repp, 1995), that if listeners were asked to identify the position of an interonset interval (IOI) between tones that was slightly longer than the others, correct detection had a negative relationship with lengthening of those beat periods in an expressive performance. If an interval was played longer in performance, listeners were not likely to report hearing that interval as longer, either as correct detection or as false alarm. These relationships are reversed if listeners are asked to detect shorter rather than longer intervals (Repp, 1998). These results were consistent with the compensation hypothesis.

However, there exists a body of empirical results indicating that listeners have a tendency to hear perceptual groups as they expect them to be-that is, separated from each other in time and initiated by sounds that are distinctive in terms of such qualities as intensity and pitch (Povel \& Okkerman, 1981; Tekman, 1995, 1997, 1998; Thomassen, 1982; Woodrow, 1909). One line of research that was specifically concerned with the perception of timing in tone sequences (Fitzgibbons, Pollatsek, \& Thomas, 1974; Thorpe et al., 1988) revealed that adults and preschool children were better at detecting a longer interval between elements of one perceptual group than at detecting a similar interval between two perceptual groups. Thorpe, Trehub, and their colleagues also found that infants could reliably make the distinction only if the longer interval appeared within a perceptual group. They attributed these findings to pattern completion, which distorted perception of between-group intervals. They argued that standard between-group intervals are hard to distinguish from longer between-group intervals because they are perceived as being longer even when they do not deviate from other intervals.

In a set of related experiments, Tekman (1997) used accented tones to create a sense of grouping. Accented tones were tones that gave emphasis to any point of time by virtue of their timing, intensity, or pitch. The specific dimension that resulted in this emphasis was not critical, and different combinations of the above-named factors could create it (Tekman, 1995). Although accenting and grouping are generally considered independent of each other (Lerdahl \& Jackendoff, 1983), other things being equal, higher intensity tones typically initiate perceptual groups (Handel, 1989, pp. 386-389). Tekman (1997) found that increasing the intensity of some of the tones resulted in a bias to report that the tones were separated from preceding ones by unequal time intervals. This effect was larger if longer silent intervals preceded the higher intensity tones than if shorter intervals preceded them. This is consistent with the earlier findings (Thorpe \& Trehub, 1989; Thorpe et al., 1988; Woodrow, 1909), in that time intervals preceding higher intensity tones were perceived as longer.

If listeners perceptually complete the missing features of a pattern, they should indeed have low sensitivity to expected variations, because they hear them as present even when they are absent. Reduced sensitivity has been observed for the presence or absence of phonemes and tones masked by noise when they were incorporated in words and chords, respectively (DeWitt \& Samuel, 1990; Samuel, 1981). This explanation implies that listeners respond to a more abstract sense of grouping in the stimulus, rather than comparing stimuli in terms of exactly how long the IOIs were heard, which is similar to Repp's (1992a) interpretation that, in phonemic restoration, the restored phonemes are not necessarily "heard" as sounds but their mental representations are activated.

One can debate the conclusion of Thorpe, Trehub, and their colleagues (Thorpe \& Trehub, 1989; Thorpe et al., 1988). Repp (1992b, 1998) would argue that the intervals separating two groups were expected to be longer and they were not detected as being longer than the standard when this expectation was fulfilled. Lower sensitivity for expected variations is not conclusive evidence that listeners hear perceptual groups as being separated by longer time intervals, as they are expected to be. If listeners compensate for their expectations, they should have low sensitivity to longer intergroup intervals, because that fits their expectations exactly. Thus, just observing low sensitivity for expected variations (Fitzgibbons et al., 1974; Thorpe et al., 1988) does not clarify which was the case.

\section{A Signal Detection Approach}

In order to resolve this ambiguity, the signal detection method was used in the present experiments. This method permits one to calculate separate measures of sensitivity and response bias or criterion. It should be noted that the response bias that is calculated using signal detection theory is quite distinct from a perceptual bias that may cause the distorted perception of temporal deviations. Perceptual bias, in the sense that Repp (1992b, 1998) uses it, is an asymmetric effect on the criterion to report timing variations in expected and unexpected directions. According to the pattern completion hypothesis, the perceptual bias is in the direction of a more liberal response criterion for expected variations than for unexpected deviations. According to the compensation hypothesis, the perceptual bias is just in the opposite direction: The criterion for reporting unexpected deviations is more liberal than the criterion for reporting expected deviations. 
From the pattern completion viewpoint, Thorpe, Trehub, and their colleagues (Thorpe \& Trehub, 1989; Thorpe et al., 1988) also argue that sensitivity should be lower for expected deviations, because they are perceived as present even when they are physically absent. Repp (1998) believes that perceptual bias is not related to sensitivity. His measure of sensitivity, which was the average of correct detection for deviations in the expected and the unexpected directions, was not significantly correlated with performance-based expectancies. However, Repp's measure of sensitivity is an unusual one. Typically, hit and false alarm rates for the same kind of stimuli are used to calculate sensitivity.

As was described above, Repp (1998) did not find significant variations in his measure of sensitivity as a function of expectations. He did verify predictions of the compensation hypothesis with two alternative measures of perceptual bias. He (Repp, 1992b, 1998) observed a negative correlation between false alarms and expressive variations in performance. That is, listeners were not likely to report hearing a normal IOI as longer if that IOI was likely to be lengthened in performance. False alarm profiles for shortening of IOIs, on the other hand, were negatively correlated with the false alarm profiles for lengthening (Repp, 1998). As a second way of measuring perceptual bias, Repp (1998) looked at the difference between correct detection of deviations in expected and unexpected directions. This measure of perceptual bias was significantly correlated with expressive timing variations in performance. Thorpe, Trehub, and colleagues (Thorpe \& Trehub, 1989; Thorpe et al., 1988) reported lower sensitivity only for expected deviations. They did not report data on deviations in the unexpected direction or response bias.

The primary goal of the present research project was to systematically examine the patterns of sensitivity and response bias in detecting timing variations, using signal detection theory. By quantifying sensitivity and bias effects for deviations in the expected and unexpected directions, it would be possible to compare these effects with the predictions from the pattern completion and compensation hypotheses. For this purpose, the signal detection procedure used by Tekman (1998) was used to discover the effects of intensity and pitch accents on the detection of timing variations. The stimuli used in the experiments reported were simple sound sequences with minimal musical structure. Thus, any effects observed would result from factors that can be generalized to auditory perception, rather than to cognitive processing of musical structure.

\section{EXPERIMENT 1}

In Experiment 1, the effect of intensity accents on the perception of variations in IOIs was investigated. The design involved factorial combination of accenting with two other independent variables. First, a sequence could contain IOIs that were either longer than the standard or shorter than the standard. Second, the deviant time intervals were distributed regularly or irregularly. This variable was used because regularity was expected to strengthen expectancies based on rhythmic structure.

In order to examine the patterns of sensitivity and response criterion for temporal variations in expected and unexpected directions, one has to determine which variations are expected on the basis of how similar sound sequences are usually heard. People asked to produce sequences of taps with intensity accents on some of the taps made the pauses coming before the accented taps shorter when the IOI for consecutive tones was $600 \mathrm{msec}$ (Billon \& Semjen, 1995; Semjen \& Garcia-Colera, 1986), but longer with a much faster tempo (IOI $=150 \mathrm{msec}$ ). In piano performance, Repp (1992b, 1998) reliably observed negative correlations between the dynamics of notes and the duration of the preceding IOIs. That is, shorter time intervals tended to precede higher intensity tones in performance. This was partly because performers played the middle portion of phrases faster and with higher intensity and partly because melody notes, which tend to be played louder and earlier, alternated with accompaniment notes. It is possible that the reverse effect that Semjen and Garcia-Colera observed with faster tempi occurred because accented taps required lifting the hand higher and this caused longer IOIs before accented taps. Such an effect that results from a motor constraint may not be related to perception. In the light of these results, listeners should have expected shorter intervals before higher intensity tones.

Hence, the pattern completion hypothesis predicts that the IOI preceding an intensity accent will be heard as shorter, and the compensation hypothesis predicts that such an interval will be heard as longer. According to the pattern completion hypothesis, intensity accents should make the response criterion for reporting shorter IOIs more liberal than the response criterion for reporting longer IOIs. Conversely, the compensation hypothesis leads to the prediction that intensity accents should make the response criterion more liberal for longer than for shorter IOIs. In the experiment, sensitivity and response criteria for temporal deviations in different directions were assessed by presenting deviations in only a single direction in each signal detection session and assuming that false alarms made in that session were false alarms for deviations in that direction.

\section{Method}

Participants. Twenty-four undergraduates of the Middle East Technical University took part in the experiment. Some of the participants received extra credit in introductory psychology courses they were enrolled in. Others were volunteers recruited through posters placed at central locations on the campus. All the participants reported having normal hearing.

Stimuli. The participants listened to pure tone sequences. The standard unaccented version of the sequences consisted of 12 tones. Each tone had a duration of $210 \mathrm{msec}$, including a $10-\mathrm{msec}$ rise 
time and a 10-msec decay time. The tones were separated by silent intervals of $50 \mathrm{msec}$, resulting in IOIs of $260 \mathrm{msec}$. Consecutive tones in the sequences had a pitch interval of one semitone (a frequency factor of $2^{1 / 12}$ ). Both ascending and descending versions of the sequences were used. The ascending sequences started on one of the chromatic pitches within the range from $\mathrm{C}_{4}(262 \mathrm{~Hz})$ to $\mathrm{F}_{4}$ $(350 \mathrm{~Hz})$. The descending sequences started on one of the chromatic pitches within the range from $\mathrm{C}_{5}(524 \mathrm{~Hz})$ to $\mathrm{F}_{5}(700 \mathrm{~Hz})$.

Accented versions of the sequences were prepared by increasing the intensity of 4 of the 12 tones by $4.5 \mathrm{~dB}$. In the regular version of the accented sequences, 2 consecutive tones with higher intensity always had 2 tones with the standard intensity between them. This resulted in a triple rhythm. In these sequences, each one of the first 3 tones in the sequence had an equal probability of being an accented tone. In the random version of the accented sequences, the 4 tones in a sequence were selected randomly to be the accented tones, with the restriction that they would not make up a triple rhythm.

The signal detection task also required sequences in which the IOIs were unequal. In these sequences, four IOIs deviated from the rest. The first tone in a sequence was considered to have an interval before it as well. If this imaginary interval was selected as one of the deviant intervals, that sequence actually had three deviant intervals. In some of the sequences, these deviant IOIs were made to be $285 \mathrm{msec}$ long by adding $25 \mathrm{msec}$ to the silent interval following the tone. These were said to have positive deviations. In other sequences, the deviant IOIs were $235 \mathrm{msec}$ long as a result of removing $25 \mathrm{msec}$ from the silent interval. These were said to have negative deviations. In the accented sequences, the deviant intervals preceded each one of the tones with higher intensity. The unaccented sequences also had regular and random versions, depending on the distribution of the deviant intervals. In the regular sequences, each consecutive pair of deviant intervals was separated by two standard intervals. In the random sequences, four intervals were selected randomly to be the deviant intervals, with the restriction that they would not make up a regular pattern.

In creating the positive and negative deviations, the duration of the tones were kept constant, whereas the IOIs were lengthened or shortened, respectively. Thus, duration of the IOIs was perfectly correlated with the duration of the interstimulus intervals (ISIs). ISIs are the time intervals between the termination of one stimulus and the beginning of the subsequent stimulus. The participants could make the distinctions they were asked to make by comparing the ISIs or, more likely, by comparing the IOIs. In earlier work, one strategy has been to leave no pauses between tones so that the duration of the sounds was equal to the IOIs (Repp, 1992b, 1998). If the results replicate the findings of this line of research, that will show that the IOI, rather than the duration of the sounds, is important in making the judgments. An alternative approach has been to use very brief sounds to mark the beginning and end of much longer time intervals. In the present project, the duration of the sounds was almost equal to the IOI, in order to make the stimuli more similar to music. The duration of the tones themselves was also selected as a representative value of note durations in a sample of Western music (Fraisse, 1956).

Apparatus. The participants were placed in a sound-attenuated booth during the experiment. Creation and presentation of the stimuli and recording of the responses were controlled by an IBMcompatible computer equipped with a Creative SoundBlaster 16 Value sound card. The participants heard the stimuli through a Technics SU-V300 amplifier and Telephonics TDH-39P earphones.

Procedure. Each participant took part in eight experimental signal detection sessions. The experimental sessions were preceded by two practice sessions. Each session consisted of 72 trials. In each trial, one sequence was heard. In 36 trials of a session, the duration of all the silent intervals in the sequence were the same. In the remaining 36 trials, the sequence contained deviant intervals. Se- quences with and without deviations in a session were ordered randomly. The beginning pitch and the direction of pitch change in the sequence varied from trial to trial, but all the sequences were similar in terms of accenting, direction of deviation, and regularity within a single experimental session. The task of the participants was to determine whether the durations of all the time intervals separating consecutive tones were the same or not for each sequence. The participant initiated the trials by pressing any one of the keys on the computer keyboard. The participants responded by pressing one of two keys assigned to the same and different responses, respectively. Feedback about the accuracy was given visually on the computer monitor after each response.

The eight experimental sessions were created by factorial combinations of three variables: (1) The sequences were either unaccented or accented; (2) in cases of unequal IOIs, the deviations were either positive or negative; and (3) the deviant intervals were arranged regularly or randomly. The deviant intervals and the higher intensity tones were coupled so that when they were present in the same sequence, each deviant interval preceded a higher intensity tone. In each experimental session, the sequences with equal IOIs and unequal IOIs were similar in all other respects. The order of the eight experimental sessions was changed from participant to participant according to a Latin-square design.

Each one of the two practice sessions also consisted of 72 trials. One practice session included unaccented sequences only, and the other included accented sequences only. All the variations of these two types of sequences were sampled with equal probability in the two respective practice sessions. The number of sequences with equal intervals and the number of sequences with unequal intervals were equal in each practice session.

\section{Results}

Sensitivity and response criterion were calculated for each one of the eight sessions for each subject. A different response to a sequence with unequal intervals was defined as a hit and a different response to a sequence with equal intervals was defined as a false alarm. Because there were some participants who did not have any misses or any false alarms in at least one session, 0.5 was added to the number of hits and false alarms, respectively, in each session. The numbers of trials with and without deviations in each session was also increased by 1 in calculating the probabilities of hits and false alarms. This adjustment should not create a large biasing effect, given the number of trials in each session (Miller, 1996). Sensitivity was calculated as the standard score corresponding to the probability of false alarms subtracted from that corresponding to the probability of hits (Macmillan \& Creelman, 1991, p. 9).

Response criterion was calculated as the negative of the average standard scores for hits and false alarms (Macmillan \& Creelman, 1991, p. 33). Because this was a measure of the criterion for responding different, lower criteria corresponded to greater bias for responding different, and higher criteria corresponded to lesser bias for responding different. Thus, positive criteria corresponded to a greater frequency of same responses, and negative criteria corresponded to a greater frequency of different responses.

Separate analyses of variance (ANOVAs) were performed on sensitivities and criteria. All three independent variables had significant effects on sensitivity. Sen- 

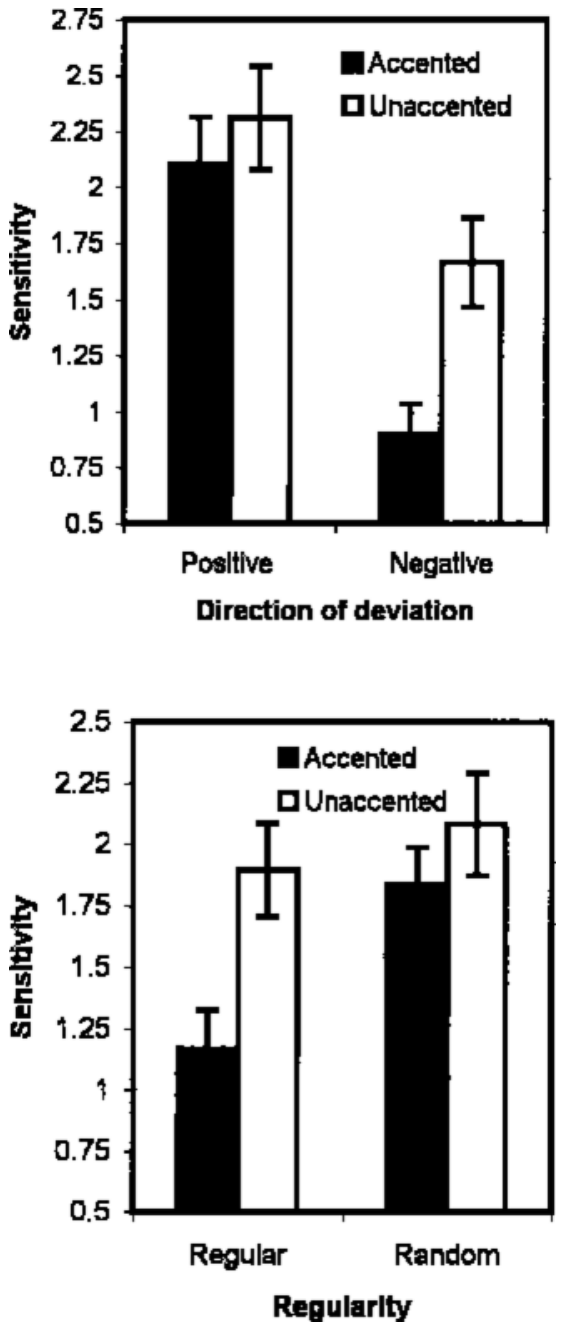

Figure 1. Mean sensitivity for positive and negative (top panel) and for regular and random (bottom panel) deviations in accented and unaccented sequences in Experiment 1. Error bars show the standard errors of the means.

sitivity was higher for random (1.96) than for regular (1.53) sequences $\left[F(1,23)=69.78, M S_{\mathrm{e}}=0.13, p<.001\right.$; eta squared $\left.\left(\eta^{2}\right)=.752\right]$, higher for unaccented (1.99) than for accented $(1.5)$ sequences $\left[F(1,23)=16.42, M S_{\mathrm{e}}=\right.$ $\left.0.7, p<.001 ; \eta^{2}=.417\right]$, and higher for positive $(2.21)$ than for negative $(1.28)$ deviations $[F(1,23)=32.87$, $\left.M S_{\mathrm{e}}=1.25, p<.001 ; \eta^{2}=.588\right]$.

As can be seen in the top panel of Figure 1, the reduction in sensitivity in accented, as compared with unaccented, sequences was greater for negative than for positive deviations $\left[F(1,23)=10.63, M S_{\mathrm{e}}=0.35, p<.005\right.$, and $\eta^{2}=.316$, for the interaction]. Detecting the variation was most difficult when a shorter interval preceded a higher intensity tone-that is, in accented sequences with negative deviations. There was also an interaction of the effects of regularity and accenting, which can be seen in the bottom panel of Figure 1. Accenting reduced sensitivity in regular sequences much more, as compared with unaccented sequences $\left[F(1,23)=20.89, M S_{\mathrm{e}}=\right.$ $\left.0.14, p<.001 ; \eta^{2}=.476\right]$. The interaction of regularity and direction of deviation $[F(1,23)=1.17, p>.05]$ and the three way interaction $[F(1,23)=2.19, p>.05]$ did not reach significance. Thus, the effects of intensity accents on the detection of positive and negative deviations did not depend on their regularity.

All three independent variables had significant effects on the criterion as well. The criterion became more liberal-that is, there was a greater tendency to respond different, for random $(0.108)$ than for regular $(0.215)$ sequences $\left[F(1,23)=8.91, M S_{\mathrm{e}}=0.06, p<.01 ; \eta^{2}=.279\right]$, for accented $(0.08)$ than for unaccented $(0.243)$ sequences $\left[F(1,23)=11.99, M S_{\mathrm{e}}=0.11, p<.005 ; \eta^{2}=.343\right]$, and for positive $(0.08)$ than for negative $(0.243)$ deviations $\left[F(1,23)=19.20, M S_{\mathrm{e}}=0.07, p<.001 ; \eta^{2}=.455\right]$.

The effect of accenting on the criterion was greater for positive than for negative deviations $[F(1,23)=13.87$, $\left.M S_{\mathrm{e}}=0.03, p<.001 ; \eta^{2}=.376\right]$. As can be seen in the top panel of Figure 2, for accented sequences with positive deviations, there was a slight bias in the direction of giving more different responses than same responses. In contrast, for accented sequences with negative deviations, response bias was in the direction of giving more same responses than different responses. The juxtaposition of shorter silent intervals with the higher intensity tones that followed them resulted in a sense of an even progression in the sequence. The average criterion for unaccented sequences was rather conservative-that is, more same than different responses, for both positive and negative deviations. The interaction of regularity with accenting $[F(1,23)=0.01, p>.05]$, regularity with direction of deviation $[F(1,23)=1.16, p>.05]$, and the threeway interaction $[F(1,23)=0.51, p>.05]$ were not significant.

\section{Discussion}

To summarize, it was found that the addition of the higher intensity tones reduced sensitivity, especially if the deviant intervals were shorter (negative deviations). The presence of higher intensity tones preceded by shorter than normal IOIs was not easy to notice as an inequality of the IOIs. It was as if these IOIs were heard as equal to the normal IOIs, which were longer in reality. The addition of the higher intensity tones also made the criterion for responding that there were unequal intervals more liberal. This effect was more pronounced when the deviant intervals were longer (positive deviations). These effects did not depend on regular placement of the higher intensity tones.

The effects of intensity accents on response criteria were consistent with the prediction from the compensation hypothesis that listeners would expect the IOIs preceding higher intensity tones to be shorter and would hear them as longer. The interaction of the effects of accenting and direction of deviation on sensitivity can be interpreted as consistent with hearing these IOIs as longer as 

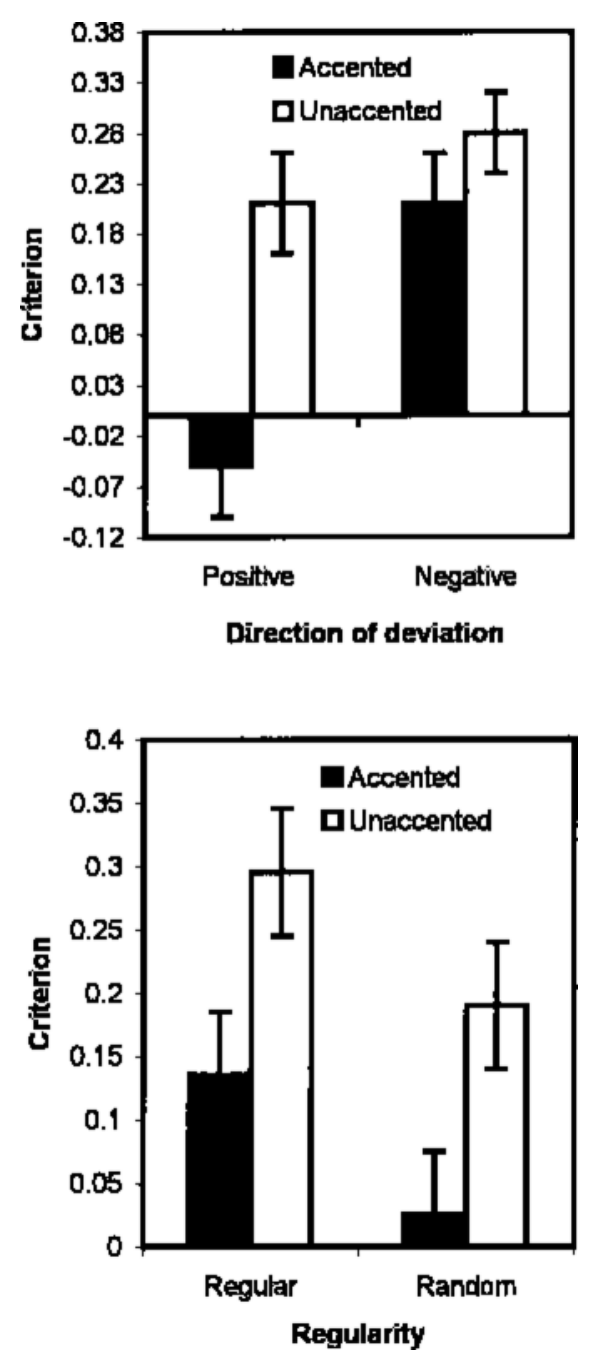

Figure 2. Mean criteria for positive and negative (top panel) and for regular and random (bottom panel) deviations in accented and unaccented sequences in Experiment 1. Error bars show the standard errors of the means.

well. In the sessions with accented sequences and negative deviations, the stimuli had either temporal regularity, which would be heard as small positive deviations, or negative deviations, which would be heard as close to regularity. In this case, the listener faced the difficulty that the sequences that were heard as being closer to having regular timing were actually the ones that contained temporal deviations. In addition, both kinds of stimuli in these sessions were close to temporal regularity. Regular timing may have acted as a perceptual magnet and made the stimuli difficult to distinguish. That is, good examplars of regular timing may have been perceived as more similar to each other than poor exemplars. Such perceptual magnet effects have been observed in signal detection tasks with speech stimuli (Iverson \& Kuhl, 1995).
In earlier research, listeners' temporal variability ratings of accented sequences with no temporal deviation and with small negative deviations, which were of the same order as those used in this experiment, were found to be very similar (Tekman, 1997). Accented sequences with positive deviations of the same magnitude were much more likely to be rated as departing from temporal regularity. Considering that sequences with equally salient rhythmic structures were found to be difficult to distinguish from each other (Tekman, 1995), if IOIs preceding intensity accents were heard as longer, shorter IOIs preceding intensity accents would be difficult to distinguish from regular IOIs.

It should be noted that although there was a significant main effect of regularity and a significant interaction involving regularity and accenting, regularity did not interact with the direction of deviation. This means the perceptual distortion was independent of the presence of a regular rhythm in the sequences. This supports the possibility that the perceptual distortion that is observed is a general property of auditory perception and that processing musical structure is not a necessary part of it.

\section{EXPERIMENT 2}

In Experiment 2, the same procedure was used to determine the effects of pitch accents. The participants listened to sequences of pure tones that either moved by semitone steps all along (unaccented sequences) or had jumps of three semitones alternating with no change in pitch (accented sequences). Jumps in pitch initiate perceptual groups, and the tones following the skips are heard as accented (Huron \& Royal, 1996; Thomassen, 1982). Earlier research has also indicated that detecting deviations in IOIs is more difficult when the IOIs correspond to pitch jumps that separate two groups of different pitches than when they do not separate perceptual groups (Drake, 1993; Thorpe \& Trehub, 1989; Thorpe et al., 1988).

Drake and Palmer (1993) found that melodically accented notes were separated from the preceding notes by longer IOIs in the performance of simple sequences and real music. Penel and Drake (1998) replicated the finding in cases in which pitch jumps did not coincide with conflicting structural features. Repp (1992b) did not find a consistent relationship between pitch change and timing in musical performance. This may be because the effect of pitch on timing may be rather weak and easily overcome by conflicting rhythmic or metric information. In the sequences that were used in the present experiment, pitch jumps did not conflict with any other structural feature. Thus, it was assumed that listeners would expect longer time intervals to precede the pitch accents.

According to the pattern completion hypothesis, IOIs preceding intensity accents should be perceived as longer. Hence, the response criterion for reporting shorter IOIs 

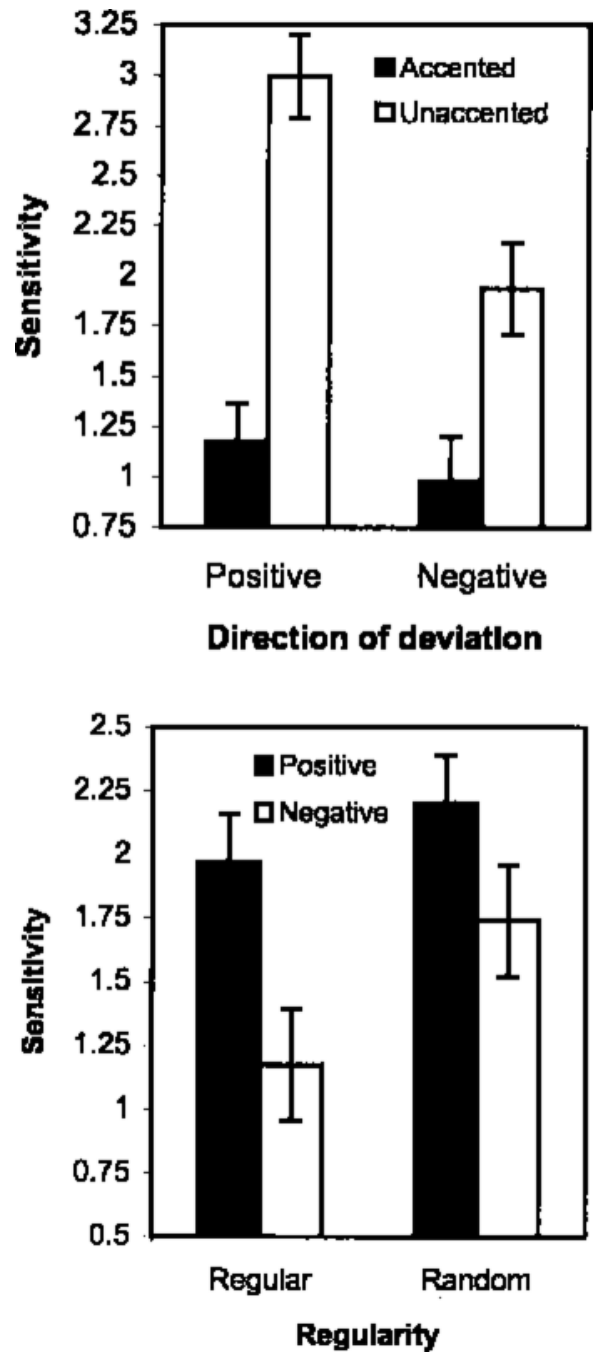

Figure 3. Mean sensitivity for positive and negative (top panel) and for regular and random (bottom panel) deviations in accented and unaccented sequences in Experiment 2. Error bars show the standard errors of the means.

should be more liberal than the response criterion for reporting shorter IOIs. According to the compensation hypothesis, these intervals should be perceived as shorter. Hence, the response criterion should be more liberal for longer than for shorter IOIs.

The task in the experiment was to detect variations in the IOIs in a sequence. The detection of slightly longer or slightly shorter intervals was compared for the cases in which they coincided with the three semitone jumps and those in which they were in unaccented sequences. Regularity was another independent variable, as in Experiment 1.

\section{Method}

Participants. Twenty-four undergraduates of Middle East Technical University, who had not participated in Experiment 1, were the participants. They were recruited in the same manner as in Experiment 1 .
Stimuli. The stimuli were prepared in the same manner as in Experiment 1, except for the method of accenting. In Experiment 2, the unaccented sequences were ascending and descending chromatic sequences. In the accented sequences, pitch changed by an interval of three semitones before 4 of the 12 tones. There were no pitch changes at other locations. In the regular sessions, these pitch jumps were placed before every 3rd tone in a sequence. In the random sessions, the positions of the pitch jumps were randomly selected. The procedure was the same as that in Experiment 1.

\section{Results}

The data from Experiment 2 were analyzed in the same way as those from Experiment 1. As in Experiment 1, sensitivity was higher for random (1.97) than for regular $(1.57)$ sequences $\left[F(1,23)=20.94, M S_{\mathrm{e}}=0.37\right.$, $\left.p<.001 ; \eta^{2}=.477\right]$, for unaccented (2.47) than for accented $(1.08)$ sequences $\left[F(1,23)=130.41, M S_{\mathrm{e}}=0.71\right.$, $\left.p<.001 ; \eta^{2}=.85\right]$, and for positive (2.09) than for negative $(1.46)$ deviations $\left[F(1,23)=16.93, M S_{\mathrm{e}}=1.12, p<\right.$ $\left..001 ; \eta^{2}=.424\right]$. Unlike Experiment 1 , however, the effect of accenting was larger for positive deviations than for negative deviations $\left[F(1,23)=27.07, M S_{\mathrm{e}}=0.33, p<\right.$ $\left..001 ; \eta^{2}=.541\right]$. That is, sensitivity in detecting longer IOIs declined by a large amount if the interval in question was juxtaposed with a large pitch interval, but it declined by a smaller amount if a shorter IOI was juxtaposed with a large pitch interval (see top panel of Figure 3). This did not appear to be a floor effect, because the standard deviations were quite uniform for the eight conditions in the experiment. (The coefficient for the correlation between condition means and standard deviations was slightly negative, $r=-$.12.) Contrary to what was observed in Experiment 1, accenting reduced the detection of positive deviations more than that of negative deviations. This meant that listeners heard the time intervals preceding the accented tones as shorter. The interaction of regularity and direction of deviation was significant in this experiment $\left[F(1,23)=5.04, M S_{\mathrm{e}}=0.27, p<.05\right.$; $\left.\eta^{2}=.18\right]$. As can be seen in the bottom panel of Figure 3, the difference between sensitivity to positive deviations and sensitivity to negative deviations was larger for regular than for random sequences. The interaction of accenting and regularity $[F(1,23)=0.64, p>.05]$ and the three-way interaction $[F(1,23)<0.01, p>.05]$ were not significant.

For the criterion, there were significant effects of regularity $\left[F(1,23)=13.77, M S_{\mathrm{e}}=0.05, p<.005 ; \eta^{2}=.374\right]$ and accenting $\left[F(1,23)=34.23, M S_{\mathrm{e}}=0.06, p<.001\right.$; $\left.\eta^{2}=.598\right]$, but the effect of direction of deviation did not reach significance $[F(1,23)=2.78, p>.05]$. As in Experiment 1 , criteria were more liberal for random $(0.07)$ than for regular (0.198) and for accented (0.033) than for unaccented (0.235) sequences. As can be seen in the top panel of Figure 4, the interaction of the effects of accenting and the direction of deviation on criterion was not reversed in Experiment 2, but the interaction that was observed in Experiment 1 did not appear $[F(1,23)=0.40$, $\left.M S_{\mathrm{e}}=0.07, p>.05\right]$. Thus, listeners were more likely to respond different to accented sequences than to unaccented sequences, but this effect did not change depend- 

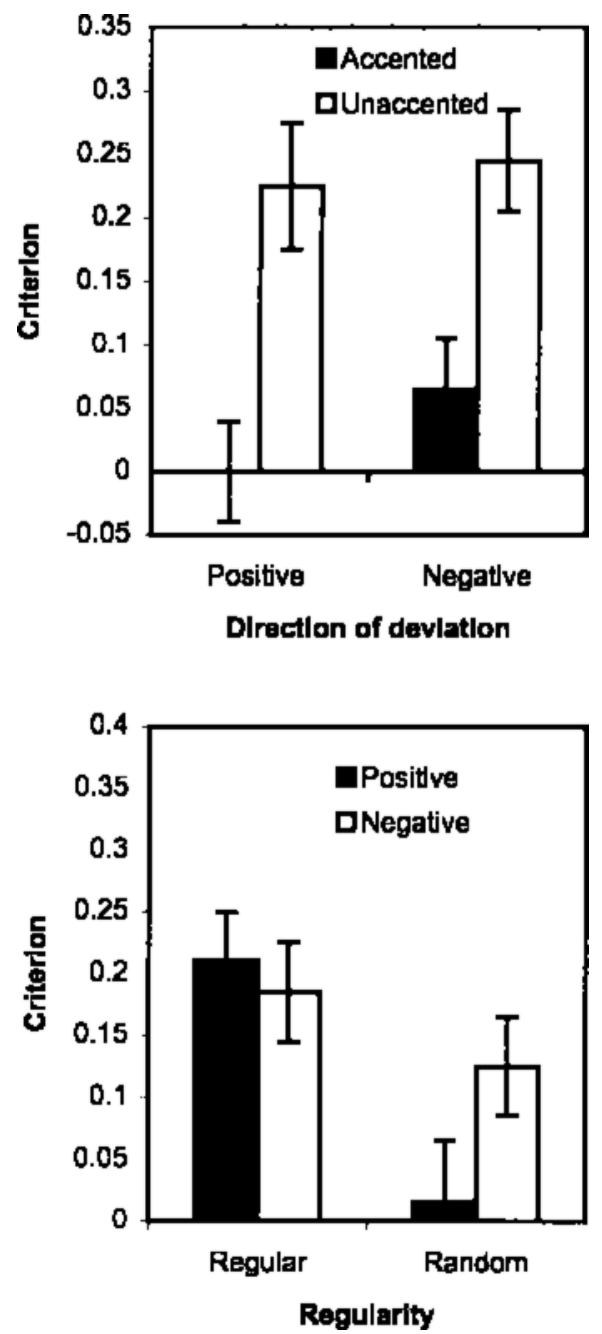

Figure 4. Mean criteria for positive and negative (top panel) and for regular and random (bottom panel) deviations in accented and unaccented sequences in Experiment 2. Error bars show the standard errors of the means.

ing on the direction of deviation. Unlike Experiment 1, combination of positive deviations with accents did not result in a large bias to respond different. The interaction of regularity and accenting $[F(1,23)=0.33, p>.05]$, regularity and direction of deviation $[F(1,23)=3.23, p>.05]$, and the three-way interaction $[F(1,23)=0.03, p>.05]$ did not reach significance.

\section{Discussion}

Some of the results of Experiment 1 were replicated in Experiment 2-namely, accenting reduced sensitivity to temporal deviations and made the response criterion more liberal (except for regular accents with positive deviations). However, accenting reduced sensitivity for detecting positive deviations more than that for negative deviations in Experiment 2. Thus, the participants easily detected shorter IOIs that accompanied jumps in pitch. A complementing pattern of average criteria was not observed: Accenting did not increase bias to report negative deviations more than positive deviations. However, the larger effect of accenting on positive deviations, which was observed in Experiment 1, was not observed in Experiment 2 . The finding that positive deviations, which would be expected in performance (Drake \& Palmer, 1993), were made difficult to detect to a greater extent, as compared with negative deviations, is just the opposite of the results of Experiment 1. As such, it is more consistent with hearing these intervals as shorter than they were, which was the prediction of the compensation hypothesis.

In Experiment 2, positive deviations, which would be expected in performance (Drake \& Palmer, 1993), were made difficult to detect to a greater extent, whereas negative deviations, which would be unexpected, were affected less. There are some findings in the literature that seem to be contradictory with this. One is the auditory kappa effect (Cohen, Hansel, \& Sylvester, 1954; Crowder \& Neath, 1995). In this effect, time intervals that correspond to larger pitch changes are perceived as longer than those corresponding to smaller pitch changes. For example, Crowder and Neath observed that listeners who were asked to judge which one of two time intervals defined by a sequence of three sounds was longer exhibited a bias to respond in favor of the time interval that corresponded to the larger pitch interval. However, in this experiment, stimuli consisted of tones that were $220 \mathrm{msec}$ long separated by silent periods ranging from 933 to $1,333 \mathrm{msec}$. These time intervals were longer than IOIs that are likely to produce a sense of sequence or stream, which is in the range of 200 to $900 \mathrm{msec}$ (Fraisse, 1963). It is doubtful whether the stimuli that were used in this experiment can tell us something about perceptual grouping in music, which was the question addressed in the present experiment.

Boltz (1998) reported another study that seems to contradict the present results. She used a task in which listeners had to judge which one of a pair of melodic sequences had a faster tempo. When sequences that included pitch accents that were created by either small or large skips in pitch were compared, listeners had a tendency to report that the one with the larger pitch skips was slower. Boltz interpreted this as meaning that the time intervals corresponding to the larger pitch skips were perceived as longer. However, the experiment does not provide direct evidence for this. Although the sequences that contained large pitch skips were perceived as slower, it is not certain that this was specifically because the time intervals corresponding to those skips were perceived as longer. Another problem common to both of these experiments is that they do not provide evidence that the way the time intervals were perceived was changed at all. The results are consistent with only a bias to respond longer or slower when large changes in pitch are present. In both of the pres- 
ent experiments, a reliable response bias to report unequal time intervals was observed when a dimension of sound other than timing was manipulated.

\section{GENERAL DISCUSSION}

The main goal of this project was to find out whether pattern completion or compensation was a better explanation of the effects of two different kinds of accenting on detection of timing variations in pure tone sequences by analyzing the pattern of sensitivity and response bias. Signal detection theory proved to be a useful tool for exploring perceptual distortions of this kind. In Experiment 1 , the pattern of performance indicated that listeners heard the IOIs preceding intensity accents as longer than other IOIs in the same sequence. Together with results showing that higher intensities and faster tempi (shorter IOIs) are positively related in performance, this result is consistent with the prediction that listeners perceptually compensated for their expectations and heard deviations from these expectations with relative ease. In Experiment 2, the results were less clear. The pattern of sensitivity pointed in the direction that IOIs coinciding with pitch skips were heard as shorter than other IOIs in the same sequence. Again, listeners were sensitive to deviations from the pattern of IOIs that was expected in performance, which was longer time intervals juxtaposed with larger pitch intervals. The results were generally consistent with the compensation hypothesis, rather than with the pattern completion hypothesis.

These results may also provide information about which level of perceptual analysis is the origin of such effects. Repp (1998) proposed three possible sources for these effects: (1) auditory processing, which includes possible psychoacoustic interactions between dimensions of sound, (2) structural perception, which includes distortions that are due to the perception of grouping structure, and (3) structural cognition, which includes expectations based on conscious interpretation of the global structure of a piece. Structural cognition is assumed to be under voluntary control, whereas auditory processing and structural perception are not (Repp, 1998). The fact that performers use some expressive variation even when they are instructed to perform mechanically (Drake \& Palmer, 1993; Gabrielsson, 1974; Penel \& Drake, 1998) indicates that structural cognition may be only partially responsible for the variations in perception and performance of music.

Because the stimuli used in the experiments did not have musical structure, the source cannot be Repp's (1998) level of structural cognition. The fact that perceptual compensation effects are observed with these stimuli indicates that, although Repp (1998) has found that structural cognition plays a role, there must be contributions from the two lower levels of processing as well. The regularity manipulation may provide information related to the distinction between auditory perception and struc- tural perception. Although irregularity of accenting does not eliminate perceptual grouping, grouping would be stronger in the regular sequences than in the random sequences. If the perceptual compensation effects were related to the level of structural perception - that is, perceptual grouping - these effects should have been larger with the regular sequences. Although there were effects of regularity, this variable did not interact with accenting in either experiment. That is, the effects of intensity and pitch accents on the perception of timing were independent of the regularity of these accents.

This finding indicates that the perceptual effects observed in these experiments are more likely to result from auditory processing - that is, the interaction of a pair of dimensions at a psychoacoustic level. This points to an interesting possibility about expressive variations in performance. It is known that some variation in the timing of notes is observed even when performers are instructed to play without any expression (Drake \& Palmer, 1993; Gabrielsson, 1974; Penel \& Drake, 1998). This may be because performers deviate from a uniform tempo, in part, because they are unable to detect these deviations. That is, rather than listeners hearing in a distorted way because of what they expect, performers may be playing in a distorted way because of the way they hear.

\section{REFERENCES}

Billon, M., \& Semjen, A. (1995). The timing effects of accent production in synchronization and continuation tasks performed by musicians and nonmusicians. Psychological Research, 58, 206-217.

Boltz, M. G. (1998). Tempo discrimination of musical patterns: Effects due to pitch and rhythmic structure. Perception \& Psychophysics, 60, 1357-1373.

Cohen, J. Hansel, C. E. M., \& Sylvester, J. D. (1954). Interdependence of temporal and auditory judgments. Nature, 174, 642-644.

Crowder, R. G., \& Neath, I. (1995). The influence of pitch on time perception in short melodies. Music Perception, 12, 379-386.

DeWitt, L. A., \& Samuel, A. G. (1990). The role of knowledge-based expectations in music perception: Evidence from musical restoration. Journal of Experimental Psychology: General, 119, 123-144.

Drake, C. (1993). Perceptual and performed accents in musical sequences. Bulletin of the Psychonomic Society, 31, 107-110.

Drake, C., \& PAlmer, C. (1993). Accent structures in music performance. Music Perception, 10, 343-378.

Fitzgibbons, P. J., Pollatsek, A., \& Thomas, I. B. (1974). Detection of temporal gaps within and between perceptual tonal groups. Perception \& Psychophysics, 16, 522-528.

Fraisse, P. (1956). Les structures rythmiques [Rhythmic structures]. Louvain: Publications Universitaires de Louvain.

Fraisse, P. (1963). The psychology of time. New York: Harper \& Row. Gabrielsson, A. (1974). Performance of rhythmic patterns. Scandinavian Journal of Psychology, 15, 63-72.

HANDEL, S. (1989). Listening: An introduction to the perception of auditory events. Cambridge, MA: MIT Press.

Huron, D., \& Royal, M. (1996). What is melodic accent? Converging evidence from musical practice. Music Perception, 13, 489-516.

IVERSON, P., \& KUHL, P. (1995). Mapping the perceptual magnet effect for speech using signal detection theory and multidimensional scaling. Journal of the Acoustical Society of America, 97, 553-562.

LERDAHL, F., \& JACKENDOFF, R. (1983). A generative theory of tonal music. Cambridge, MA: MIT Press. 
Macmillan, N. A., \& Creelman, C. D. (1991). Detection theory: A user's guide. Cambridge: Cambridge University Press.

Miller, J. (1996). The sampling distribution of $d^{\prime}$. Perception \& Psychophysics, 58, 65-72.

Penel, A., \& Drake, C. (1998). Sources of timing variations in music performance: A psychological segmentation model. Psychological Research, 61, 12-32.

Povel, D.-J., \& OK Kerman, H. (1981). Accents in equitone sequences. Perception \& Psychophysics, 30, 565-572.

REPP, B. H. (1990). Patterns of expressive timing in performances of a Beethoven minuet by nineteen famous pianists. Journal of the Acoustical Society of America, 88, 622-641.

Repr, B. H. (1992a). Perceptual restoration of a "missing" speech sound: Auditory induction or illusion? Perception \& Psychophysics, 51, 14-32.

REPP, B. H. (1992b). Probing the cognitive representation of musical time: Structural constraints on the perception of timing perturbations. Cognition, 44, 241-281.

REPP, B. H. (1995). Detectability of duration and intensity increments in melody tones: A partial connection between music perception and performance. Perception \& Psychophysics, 57, 1217-1232.

REPP, B. H. (1996). The art of inaccuracy: Why pianists' errors are difficult to hear. Music Perception, 14, 161-184.

REPP, B. H. (1998). Variations on a theme by Chopin: Relations between perception and production of timing in music. Journal of Experimental Psychology: Human Perception \& Performance, 24, 791-811.

Samuel, A. G. (1981). Perceptual restoration: Insights from a new methodology. Journal of Experimental Psychology: General, 110, 474-494.
Semjen, A., \& Garcia-Colera, A. (1986). Planning and timing of finger-tapping sequences with a stressed element. Journal of Motor Behavior, 18, 287-322.

Shaffer, L. H., Clarke, E. F., \& Todd, N. (1985). Meter and rhythm in piano playing. Cognition, 20, 61-77.

Tekman, H. G. (1995). Cue trading in the perception of rhythmic structure. Music Perception, 13, 17-38.

Tekman, H. G. (1997). Interactions of perceived intensity, duration, and pitch in pure tone sequences. Music Perception, 14, 281-294.

Tekman, H. G. (1998). Effects of melodic accents on perception of intensity. Music Perception, 15, 391-401.

Thomassen, J. M. (1982). Melodic accents: Experiments and a tentative model. Journal of the Acoustical Society of America, 71, 15691604.

Thorpe, L. A. \& Trenub, S. E. (1989). Duration illusion and auditory grouping in infancy. Developmental Psychology, 25, 122-127.

Thorpe, L. A., Trehub S. E., Morrongiello, B. A., \& Bull, D. (1988). Perceptual grouping by infants and preschool children. Developmental Psychology, 24, 484-491.

TodD, N. P. (1985). A model of expressive timing in tonal music. Music Perception, 3, 33-58.

Windsor, W. L., \& Clarke, E. F. (1997). Expressive timing and dynamics in real and artificial musical performances: Using an algorithm as an analytical tool. Music Perception, 15, 127-152.

Woodrow, H. (1909). A quantitative study of rhythm. Archives of Psychology, 14, 1-66.

(Manuscript received December 2, 1998; revision accepted for publisher June 5, 2000.) 